\title{
Nivel de calidad de vida del adulto mayor del centro gerontológico de Tetepango Hidalgo, pre y post intervenciones lúdicas
}

Life quality level of the elderly adult of the Gerontological center of Tetepango Hidalgo, pre and post ludic interventions

\section{Benjamín López Nolasco ${ }^{a}$, Ingrid L. Álvarez Juárez ${ }^{b}$, Zumico Ruíz Hernández $^{c}$, Lizeth Vázquez Hernández $^{c}$, Ariana Maya Sánchez ${ }^{d}$, Edith A. Cano Estrada ${ }^{e}$}

\begin{abstract}
:
This research aimed to: Analyze the level of quality of life in the elderly of the gerontological center of Tetepango Hidalgo, before and after playful studies. Method: quantitative approach, with an applied applicability, level of correlation scope, quasi-experimental design, pro-lective, longitudinal, prospective, 14 older adults were involved. Materials: the theory of the adaptation model of Sr. Callista Roy will be seen as a reference, the WHOQOL-BREF quality of life scale was applied in two moments in the sample. Results: 14 older adults participated, predominantly female (100\%), domestic occupation (100\%), incomplete primary school (57.1\%), married marital status (50.0\%), more frequent pathologies were (42.9\%) more than Two chronic degenerative diseases. an increase in physical health (56.8\%), psychological health (49.7\%) in social health (49.7\%) and environmental health (49.7\%) was increased. Discussion: the study was feasible the application of recreational interventions, in the Gerontological center of Tetepango Hidalgo. Conclusion: The quality of life depends on the lifestyle that will take from birth, in order to age with health, the living conditions of the population must be improved.
\end{abstract}

\section{Keywords:}

Quality of life, elderly, playful patients

\section{Resumen:}

Esta investigación tuvo como objetivo: Analizar el nivel de calidad de vida en el adulto mayor del centro gerontológico de Tetepango Hidalgo, pre y post intervenciones lúdicas. Método: enfoque cuantitativo, con una aplicabilidad aplicada, nivel de alcance correlacionar, diseño cuasi experimental, pro lectivo, longitudinal, prospectivo, se contó con la participación de 14 adultos mayores.

Materiales: se tomó como referente la teoría de Modelo de adaptación de Sor Callista Roy, se aplicó en dos momentos la escala de calidad de vida WHOQOL-BREF en la muestra. Resultados: participaron 14 adultos mayores, predomino el sexo femenino (100\%),

\footnotetext{
a Autor de Correspondencia, Universidad Autónoma del Estado de Hidalgo, Escuela Superior de Tlahuelilpan, Profesor Investigador, Email: lopez8496@uaeh.edu.mx

${ }^{\mathrm{b}}$ Universidad Autónoma del Estado de Hidalgo, Escuela Superior de Tlahuelilpan, Alumno de la Licenciatura en Enfermería, Email: 339756@uaeh.edu.mx

c Universidad Autónoma del Estado de Hidalgo, Escuela Superior de Tlahuelilpan, Alumno de la Licenciatura en Enfermería, Email: 339852@uaeh.edu.mx

d Universidad Autónoma del Estado de Hidalgo, Escuela Superior de Tlahuelilpan, Profesor por asignatura de la Licenciatura en Enfermería, Email: arianamaya@uaeh.edu.mx

e Universidad Autónoma del Estado de Hidalgo, Escuela Superior de Tlahuelilpan, Profesor Investigador de la Licenciatura en Enfermería, Email: edithce@uaeh.edu.mx
} 
ocupación hogar (100\%), escolaridad primaria incompleta (57.1\%), estado civil casada (50.0\%), Patologías más frecuentes fueron (42.9\%) más de dos enfermedades crónico degenerativas. se observó un aumento en salud física (56.8\%), salud psicológica (49.7\%) en salud social (49.7\%) y salud ambiental (49.7\%). Discusión: el estudio fue factible la aplicación de intervenciones lúdicas, en el centro Gerontológico de Tetepango Hidalgo. Conclusión: La calidad de vida depende del estilo de vida que se lleve desde el nacimiento, para envejecer con salud se deben de mejorar las condiciones de vida de la población.

\section{Palabras Clave:}

Calidad de vida, adulto mayor, intervenciones lúdicas

\section{Introducción}

Hablar de calidad de vida refiere un concepto que hace alusión a varios niveles de generalidad desde el bienestar social o comunitario hasta ciertos aspectos específicos de carácter individual o grupal. Por lo tanto calidad de vida tiene diferentes definiciones relacionando a la salud.

Por la problemática antes mencionada surge la siguiente pregunta de investigación.

¿Cuál es el nivel de calidad de vida del adulto mayor del Centro Gerontológico de Tetepango Hidalgo, pre y post intervenciones lúdicas?

Diversos estudios han demostrado que la calidad de vida de los adultos mayores, está asociada a tener mayor edad y las condiciones sociodemográficas, esto similar a Castiblanco, et al. En su estudio Capacidad y percepción de autocuidado del adulto mayor del programa Centro día/vida, arrojo como resultado que el adulto mayor presentó adecuada capacidad y percepción de autocuidado en actividad física, alimentación, medicación, adicciones y hábitos tóxicos.(Myriam Angélica Castiblanco Amaya, 2016) Y Melgizo, Encontró en su estudio Calidad de vida en adultos mayores en Cartagena, una mayor prevalencia de apoyo social, independencia para realizar actividades básicas e instrumentales de la vida diaria, a fin de ver la calidad de vida relacionada con salud como excelente. (Estela Melguizo-Herrera, 2017)

\section{Antecedentes}

Baro, En su estudio Acciones educativas para mejorar la calidad de vida en adultos mayores, en 2017, su objetivo que elaboro fue una propuesta de acciones educativas que permitieron mejorar la calidad de vida en los adultos mayores del círculo de abuelo del Consejo Popular "Pastorita", mediante el cual utilizo un método descriptivo, prospectivo y longitudinal, arrojando resultados de edad que predominaron de 60 a 70 años con $66.6 \%$ seguido del 71 a 80 años (33.4\%) mientras que el sexo predominante fue femenino con $80 \%$, así mismo el concluyo que el envejecimiento y la vejez dependen de la calidad de vida que se lleve desde el nacimiento.

Melgizo, En su estudio Calidad de vida en adultos mayores en Cartagena, Colombia, en 2017, su objetivo fue determinar factores asociados a la calidad de vida de los adultos mayores en Cartagena, 2015,mediante un método de estudio transversal, a medida que tomó como referente la teoría de calidad de vida relacionada con salud, utilizando el test de Pfeiffer, encuesta sociodemográfica, escala de depresión de Yesavage, cuestionario de salud auto percibida, escala de Barthel, Lawton y Brody, a tal logro obtuvo como resultado alta prevalencia de apoyo social, independencia para realizar actividades básicas e instrumentales de la vida diaria, percibir la calidad de vida relacionada con salud como excelente, finalmente la calidad de vida se agrupo positivamente a percibir apoyo social adecuado y a la independencia para realizar actividades instrumentales de la vida diaria y negativamente al hecho de trabajar o tener pensión. 
Cano Gutiérrez y Carlos en su estudio evaluación de factores asociados con el estado funcional en adultos de 60 años y adultos mayores en Bogotá, Colombia, en el año 2017, donde el objetivo fue determinar y caracterizar los factores sociodemográficos y las condiciones de salud son relacionadas con el deterioro funcional en adultos mayores en Bogotá Colombia, emplea las escalas de Barthel y Lawton, mantiene como variables independientes los factores sociodemográficos, las enfermedades concomitantes, las medidas antropométricas y los terciles de actividad física, arroja que una mayor funcionalidad en actividades instrumentales así mismo se asoció con una menor edad, de igual modo se establecen múltiples factores relacionados con la alteración funcional que pueden modificarse para disminuir la dependencia en este grupo poblacional.

\section{Justificación}

Debido al aumento de la esperanza de vida y a la disminución de la tasa de fecundidad, la proporción de personas mayores de 60 años está aumentando más rápidamente que cualquier otro grupo de edad en casi todos los países. El envejecimiento de la población puede considerarse un éxito de las políticas de salud pública y el desarrollo socioeconómico, pero también constituye un reto para la sociedad, que debe adaptarse a ello para mejorar al máximo la salud y la capacidad funcional de las personas mayores, así como su participación social y su seguridad.

Según datos de la OMS entre 2015 y 2050, se determinó que la población mundial con más de 60 años de edad pasara de 900 millones hasta 2000 millones, lo que representa un aumento del $12 \%$ al $22 \%$, marcando esto un envejecimiento más acelerado que los años precedentes, es posible que disminuyan los ingresos que necesitan los países de altos ingresos para ayudar a las personas mayores a realizar actividades básicas como la comida y el aseo, sin embargo la prevalencia de limitaciones menos graves de la funcionalidad es la misma.
La Organización Mundial de la Salud define el envejecimiento activo como el proceso en que se optimizan las oportunidades de salud, participación y seguridad a fin de mejorar la calidad de vida de las personas a medida que envejecen. El envejecimiento activo permite que las personas realicen su potencial de bienestar físico, social y se centra en las personas mayores.

En México residen 119.5 millones de personas de las cuales $51.4 \%$ son mujeres y $48.6 \%$ son hombres, de acuerdo a la Encuesta Intercensal aplicada en 2015. El INEGI señalo que la transición demográfica ha provocado cambios en la población, dando lugar a procesos de envejecimiento, señalando que para el año 2030 se estima que el porcentaje de adultos mayores sea de 20.4 millones, lo que representara el 14.8 $\%$, esto con las proyecciones del Consejo Nacional de Población (CONAPO).

Dentro de las estadísticas de calidad de vida en el adulto mayor se encontró que de acuerdo con las proyecciones que estima el consejo nacional de población (CONAPO), en 2017 habitan en el país casi 13 millones de personas de 60 y más años.

Actualmente se estima que las personas en México alcancen una esperanza de vida de 75 años. El envejecimiento de la población es un reto para la sociedad, ya que se requiere mejorar al máximo la salud y la capacidad funcional de las personas mayores, así como su participación social, independencia económica, convivencia social y su seguridad.

La vejez constituye un problema social que reclama mayor atención, incrementando la demanda de servicios de salud, vivienda, pensión y espacios urbanos que permitan el tránsito de las personas, debido a que el adulto mayor experimenta cambios de funciones y condiciones, sociales, educativas, culturales, artísticas, recreativas, deportivas y productivas con los que se fomenta su organización e intervención en la solución de sus problemas. 
Dentro de las estadísticas de calidad de vida en el adulto mayor se encontró que de acuerdo con las proyecciones que estima el consejo nacional de población (CONAPO), en 2017 habitan en el país casi 13 millones de personas de 60 y más años

Es importante procurar que las personas mayores tengan una valoración positiva sobre sí mismos y sus capacidades, así como brindarles aquellos elementos que le permitan reconocer su potencial de aprendizaje, sabiduría, toma de decisiones y puedan seguir considerándose personas productivas.

\section{Objetivo}

Analizar el nivel de calidad de vida en el adulto mayor del centro gerontológico de Tetepango Hidalgo, pre y post intervenciones lúdicas.

\section{Planteamiento del problema}

Hablar de calidad de vida refiere un concepto que hace alusión a varios niveles de generalidad desde el bienestar social o comunitario hasta ciertos aspectos específicos de carácter individual o grupal. Por lo tanto calidad de vida tiene diferentes definiciones relacionando a la salud.

La calidad de vida está relacionada con lo que la persona percibe del mundo, de sus relaciones con las personas próximas y del trato que recibe por parte de todas las personas y las situaciones diversas en las que está inmersa.

El adulto mayor pasa por una etapa de la vida que se considera como la última, en la que los proyectos de vida ya se han consumado, siendo posible poder disfrutar de lo que queda de vida con mayor tranquilidad. Usualmente las personas de la tercera edad han dejado de trabajar, o bien jubilan por lo que su nivel de ingresos decrece en forma considerable, lo que junto con los problemas de salud a la edad pueden traer consecuencias en todos los ámbitos de su vida.
La falta de estimulación cognitiva interfiere negativamente en la realización de actividades diarias y capacidades mentales superiores como la memoria, juicio, razonamiento abstracto, concentración, atención y praxias. También se presentan en esta etapa de vida, problemas de salud posiblemente asociados a ciertas emociones causadas por sentimientos de abandono, soledad, aislamiento, minusvaloración, entre otros.

Las actividades lúdicas se reducen con la edad y constituyen un indicador de salud.

La reducción de actividades que incluyan un esfuerzo motor, junto a la lentitud de los reflejos, provocan descoordinación y torpeza motriz. La lúdica tiene una incidencia específica sobre el retraso de la involución de forma considerable, previniendo enfermedades y contribuyendo a mantener la independencia motora y sus beneficios sociales y afectivos.

El envejecimiento es un proceso cuya calidad está directamente relacionada con la forma, como la persona satisface sus necesidades a través de todo su ciclo vital. En las sociedades envejecen a ritmo creciente, promocionar la calidad de vida en la vejez es el reto más inmediato en la sociedad, el creciente aumento en la esperanza de vida, el descenso sin precedentes históricos de la tasa de natalidad, los cambios en la estructura en el tamaño, en las formas en la familia, los cambios en el status de las mujeres, la reducción creciente de las tasas de actividad laboral entre las personas de cincuenta y cinco años y más, han convertido el envejecimiento dentro de la sociedad en una cuestión de máximo interés.

De una forma u otra, los factores sociales e individuales relacionados con el nivel de vida, son los que determinan la calidad de vida de este grupo poblacional y mejoran en la medida que ellos como un todo funcionen y se integren.

La importancia que tiene la solución del problema es que el adulto mayor pueda vivir en más y mejores condiciones físicas, sociales y mentales, 
producto que el avance social está orientado hacia esa dirección: buscar un modelo de envejecimiento competente en un sentido útil y productivo, capaz de fortalecer su calidad de vida.

Es muy importante señalar que como sociedad, reconozcamos el papel de los adultos mayores y revalorar los grandes aportes que su experiencia y conocimientos que representan para el fortalecimiento de nuestro tejido social y de nuestra identidad como Nación.

\section{Método y materiales}

Enfoque cuantitativo, con una aplicabilidad aplicada, tiene un nivel de alcance correlacional, porque relacionara los patrones de adaptación, antes y después de la intervención, el diseño es cuasi experimental, la forma de recolección de datos es proyectivo, es longitudinal, es prospectivo.

El universo con el que se trabajó durante esta investigación, estuvo constituido por un total de 20 adultos mayores de 65 años de edad y más que acuden al centro gerontológico de Tetepango Hgo. El tipo de muestra por disponibilidad determinada, con la fórmula de poblaciones finitas, tomando en cuenta los criterios de inclusión, exclusión y eliminación, con un total de 14 adultos mayores. Para el análisis de los datos de la investigación se utilizó el paquete estadístico SPSS versión 25 , se aplicó tanto estadística, descriptica como interferencial, se obtuvieron medidas de resumen, tendencia central y variabilidad.

Criterios de inclusión:

- Adultos mayores de ambos sexos.

- Que estén entre la edad entre 65 - 70 años.

- Adultos mayores que quieran participar en el estudio.

- Adultos mayores inscritos al centro gerontológico de Tetepango Hidalgo.
- Adultos mayores que firmen el consentimiento informado.

El instrumento que se aplicó para medir la variable planteada, fue la Escala de calidad de vida WHOQOL-BREF proporciona un perfil de calidad de vida, dando una puntuación global de calidad de vida, de las áreas y de las facetas que lo componen, integrado por un total de 26 preguntas, dos preguntas globales: calidad de vida global y salud general. Cada ítem tiene 5 opciones de respuesta ordinales tipo Likert y todos ellos producen un perfil de cuatro áreas: salud física, psicológica, relaciones sociales y ambiente (Badía Xavier), el alpha de cronbach para la escala total es de .89 .

Se consideraron los aspectos éticos contemplados en la Declaración de Helsinki de la asociación médica mundial (CONAMED, s.f.) Y el Reglamento de la Ley General de Salud en materia de investigación para a salud. (SALUD, 2014)

\section{Resultados}

Las variables sociodemográfica observan, una población total de 14 adultos mayores, correspondientes al $100 \%$, de esta cifra se desprende una f de 14 equivalente al $100.0 \%$ que son de sexo femenino, del total de la población con f de 1 y $7.1 \%$, se representan las edades de $61,68,69,71,76,77,80,83$, con $f$ de y $14.3 \%$, se representan las edades de $63,65,78$, de esta cifra se desprende una $f$ de 14 equivalente al $100.0 \%$ se dedican a labores del hogar, de la variable de escolaridad, con $f$ de 8 y $57.1 \%$ representan a primaria incompleta, con $f$ de 5 y $35.7 \%$ representan a primaria completa y con $\mathrm{f}$ de 1 y $7.1 \%$ representan a secundaria incompleta, de esta cifra se desprende una $f$ de 7 equivalente al $50.0 \%$ son casadas, con una f de 6 representando el $42.9 \%$ son viudas y de acuerdo a la última $f$ de 1 el $7.1 \%$ están en unión libre, el $92.9 \%$ de la muestra es de religión católica mientras que el $7.1 \%$ es de religión mormón, correspondiendo al $100 \%$ de la población total de la muestra, correspondiendo con un porcentaje de $14.3 \%$ 
diabetes, $14.3 \%$ hipertensión, el $42.9 \%$ refiere tener más de dos patologías y el 28,6 ninguna.

En la obtención de los resultados se observó una creciente en relación a los resultados preintervención, solo en tres casos se observó una disminución en el resultado de la primera medición.

De acuerdo al área de salud física se observa un aumento post intervención, así mismo el área psicológica, muestra un aumento, en el valor de buena aumentando la $\mathrm{f}$ a 7 , con un $\%$ de 49.7, disminuyendo el valor de buena, el cual al inicio tenia f de 9 y \% de 63.9, en el área ambiental se puede observar un notable aumento, en el valor de alta aumentando la f a 2 , con un \% de 14.2 .

\section{Discusión}

En el presente estudio fue factible la aplicación de actividades lúdicas, en el centro Gerontológico de Tetepango Hidalgo.

En cuanto al perfil sociodemográfico de los participantes de la población de estudio, se obtuvieron los siguientes resultados, la edad promedio de los adultos mayores oscila entre 6183 años, el 100\% corresponde al género femenino. Además de observar que el $100 \%$ se dedica a las labores domésticas, el $57.1 \%$ refirió tener primaria incompleta, el $50 \%$ son casadas, un $92.9 \%$ son de religión católica y el $42.9 \%$, refiere tener más de dos padecimientos.

Respeto al efecto de la intervención (actividades lúdicas) se probó estadísticamente que fue significativa, ya que los resultados arrojaron post intervención $14.2 \%$ en salud psicológica, $21.4 \%$ en el área social y en salud ambiental $7.1 \%$ en calidad de vida. Esto es similar a Melgizo, et al. En su estudio Calidad de vida en adultos mayores en Cartagena, Colombia, en 2017, cuyo objetivo fue determinar factores asociados a la calidad de vida de los adultos mayores en Cartagena, 2015, utilizando un método de estudio transversal, tomó como referente la teoría de calidad de vida relacionada con salud, empleando los test de Pfeiffer, encuesta sociodemográfica, escala de depresión de Yesavage, cuestionario de salud auto percibida, escala de Barthel, Lawton y Brody, arrojando como resultado alta prevalencia de apoyo social, independencia para realizar actividades básicas e instrumentales de la vida diaria, percibir la calidad de vida relacionada con salud como excelente, concluyendo La calidad de vida se asoció positivamente a percibir apoyo social adecuado y a la independencia para realizar actividades instrumentales de la vida diaria y negativamente al hecho de trabajar o tener pensión.

Baro, et al. En su estudio Acciones educativas para mejorar la calidad de vida en adultos mayores, en 2017, cuyo objetivo fue elaborar una propuesta de acciones educativas que permitan mejorar la calidad de vida en adultos mayores del círculo de abuelo del Consejo Popular "Pastorita", utilizando un método fue descriptivo, prospectivo y longitudinal, arrojo los resultados de edad que predomina es de 60 a 70 años con $66.6 \%$ seguido del 71 a 80 años (33.4\%) y el sexo predominante es el femenino con $80 \%$, concluyendo el envejecimiento y la vejez dependen de la calidad de vida que se lleve desde el nacimiento.

González-Celis Ana, En su estudio autoeficacia, percepción de salud y soledad, sobre la calidad de vida en adultos mayores, en 2017, cuyo objetivo fue Probar si existen diferencias entre los niveles de autoeficacia para realizar actividades cotidianas, percepción de salud y sentimientos de soledad, sobre la calidad de vida y sus dimensiones, utilizando un método no probabilístico intencional, selectivo y por participación voluntaria, consentida e informada compuesta por 149 adultos mayores, arrojando como resultado las puntuaciones de calidad de vida y su dimensión "participación social" son diferentes dependiendo del nivel de percepción de autoeficacia para actividades sociales, concluyendo que el nivel de autoeficacia para 
realizar actividades sociales tiene un impacto en la calidad de vida, ya que los adultos mayores que reportaron tener mayor autoeficacia, tienen mejor calidad de vida; por el contrario, los que tienen un nivel de autoeficacia pobre reportan que su nivel de calidad de vida es más bajo.

\section{Conclusión}

El envejecimiento depende del estilo de vida que se lleve desde el nacimiento, para envejecer con salud se deben de mejorar las condiciones de vida de la población, brindar bienestar asegurando servicios de salud integrales, desarrollar una cultura donde la vejes sea considerada como un símbolo de experiencia, sabiduría, respeto y contribuya al fortalecimiento de la solidaridad y el apoyo mutuo entre generaciones.

\section{Referencias}

1. Tania Baró. Acciones Educativas para mejorar la calidad de vida en adultos mayores. revista información científica. 2017; 96(5).

2. Estela melguizo-herrera srvr. calidad de vida en adultos mayores en Cartagena, Colombia. revista de salud pública issn 0124-0064. 2017; 19(4): p. 549-554.

3. Gonzalez-celis a. autoeficacia, percepción de salud y soledad, sobre la calidad de vida en adultos mayores. [online].; 2017 [cited 2018 September. available from: https://www.researchgate.net/profile/ana_gonza lez-

celis/publication/321447097_autoeficacia_perc epcion_de_salud_y_soledad_sobre_la_calidad de_vida_en_adultos_mayores/links/5a5ab8c6a6 fdcc3bfb5f66bd/autoeficacia-percepcion-desalud-y-soledad-sobre-la-calidad.

4. Carlos cano-gutiérrez mgbcroajarst. scielo. [online].; 2017 [cited 2018 septiembre. available from:

http://www.scielo.org.co/pdf/bio/v37s1/01204157-bio-37-s1-00057.pdf.

5. Myriam angélica castiblanco amaya, e. f. (2016 de octubre de 2016). scielo. recuperado el septiembre de 2017, de scielo: http://www.scielo.org.co/pdf/sun/v33n1/20117531-sun-33-01-00058.pdf
6. Badía xavier, s. m. (1999). "la medida de la salud. guía de escalas de medición en español". (e. edimac., editor) recuperado el septiembre de 2018, de "la medida de la salud. guía de escalas de medición en español" :www.sagg.org.ar/wp/wpcon+tent/uploads/2015/07/whoqol1.doc

7. Oms. organización mundial de la salud. [online]. mayo; 2017. available from: http://www.who.int/features/factfiles/ageing/es/

8. Oms. ecured. [online].; 2013 [cited 25052018. available from: https://www.ecured.cu/calidad_de_vida.

9. Guilca rma. "las actividades lúdicas en la calidad de vida en el adulto mayor del hogar de ancianos instituto estupiñan del cantón latacunga". investigacion. ambato - ecuador:; 2017.

10. Mayor ieypleea. users. revista killkana salud y bienestar. $2017 ;:$ p. 6.

11. Cruz mayra cnyoi. intervención educativa y programa lúdico en el adulto mayor. revista killkana salud y bienestar. 2017;: p. 6.

12. Conamed. (s.f.). recuperado el septiembre de 2018 , de http://www.conamed.gob.mx/prof_salud/pdf/he lsinki.pdf

13. Salud, 1. g. (2014). salud.gob. recuperado el 2018, de salud.gob: http://www.salud.gob.mx/unidades/cdi/nom/co mpi/rlgsmis.html

14. Iris Espinoza, P. O. (2011). Validación del cuestionario de calidad de vida ((WHOQOLBREF) en adltos mayores chilenos. Rev Med Chile, 8. 